\title{
Spall-Velocity Reduction in Double-Pulse Impact on Tin Microdroplets
}

\author{
Randy A. Meijer® ${ }_{1,2, *}$ Ruben Schupp $\odot,{ }^{1}$ John Sheilø, ${ }^{1}$ Mikhail M. Basko $\odot,{ }^{3}$ Kjeld S. E. Eikema, ${ }^{1,2}$ \\ Oscar O. Versolato ${ }^{1,}{ }^{1,2}$ and Stefan Witte ${ }^{1,2, \dagger}$ \\ ${ }^{1}$ Advanced Research Center for Nanolithography (ARCNL), Science Park 106, Amsterdam 1098 XG, Netherlands \\ ${ }^{2}$ LaserLab, Department of Physics and Astronomy, Vrije Universiteit Amsterdam, De Boelelaan 1105, \\ Amsterdam $1081 \mathrm{HV}$, Netherlands \\ ${ }^{3}$ Keldysh Institute of Applied Mathematics, Russian Academy of Sciences, Miusskaya Square 4, Moscow 125047, \\ Russia
}

(Received 30 September 2020; revised 14 July 2021; accepted 30 July 2021; published 16 August 2021)

\begin{abstract}
We explore the deformation of tin microdroplets of various diameters induced by two consecutive laser pulses having pulse durations of $0.4 \mathrm{~ns}$. Impact of laser pulses with this duration mainly leads to shockwave-induced cavitation and spallation. The main result obtained in this work is the observation of a strong reduction of the spall velocity that depends on the time delay between the two pulses. This reduction reveals a complex interplay between plasma recoil pressure and shock-wave-driven deformation, and enables an estimation of the moment of spall formation and the average shock-wave propagation velocity. We find that the shock wave traverses the droplet with an average velocity ranging from 1.2 to 1.6 times the speed of sound. We study the effects of the energy of the second pulse on the deformation and qualitatively discuss the formation of microjets. Crucially, we demonstrate the ability to manipulate the microdroplet expansion and spallation with double-pulse sequences, thereby increasing the portfolio of obtainable target shapes for droplet-based extreme ultraviolet light sources.
\end{abstract}

DOI: 10.1103/PhysRevApplied.16.024026

\section{INTRODUCTION}

The interaction of laser pulses with metallic, and specifically tin, microdroplets and the resulting deformation has been a significant field of interest since the introduction of the prepulse in current-day extreme ultraviolet (EUV) sources for nanolithography [1,2]. The purpose of the prepulse is to initiate a hydrodynamic expansion of the droplet. This deformation process is crucial, as it leads to a tin target with a more beneficial mass distribution for interaction with a following more energetic laser pulse, whose purpose is to create the optimum plasma conditions enabling EUV light generation [3].

Two main types of target are accessible using a conventional single laser pulse. Deformation into a thin liquid sheet can be initiated by impact of a laser pulse with a duration of several to tens of nanoseconds [4,5]. For metallic droplets, this type of deformation is initiated by a recoil pressure from the expanding plasma generated by the laser pulse [6], and the resulting propulsion, expansion, fragmentation, and overall target morphology are well understood [4-10]. Impact of intense pulses of shorter duration $(\lesssim 1 \mathrm{~ns})$ launches a shock wave into the droplet.

\footnotetext{
*r.meijer@arcnl.nl

†s.witte@arcnl.nl
}

Because of the spherical geometry, the shock wave converges towards the center of the droplet which leads to cavitation if a sufficiently low negative pressure is reached [11-19]. The formed vapor cavity can then rapidly expand, turning the initial droplet into a thin shell. Additionally, albeit with a laser energy threshold significantly higher than that of the central cavitation, spall will be ejected upon reflection of the shock wave from the opposing droplet surface $[13,15-18,20,21]$. Cavitation with or without spallation will lead to a single- or double-domed target morphology, respectively. Although these two types of deformation are predominantly separated into regimes by origin of the laser pulse duration, there is significant overlap and both types will often coexist. Partly due to this coexistence, a broad diversity of obtainable deformation types exists that remains largely unexplored.

Industrially relevant EUV sources typically operate with tin targets of a few hundred micrometers in diameter, formed within a few microseconds after prepulse laser impact [14,22-24]. When operating in the shock-wavedominated deformation regime using "short," subnanosecond pulses, the expansion velocities needed to reach such diameters can only be produced by pressure waves that exceed the spallation threshold $[15,17,18]$. The spall itself can however be considered unwanted, as tin fragments are propelled away at typical velocities several times the 
(a)

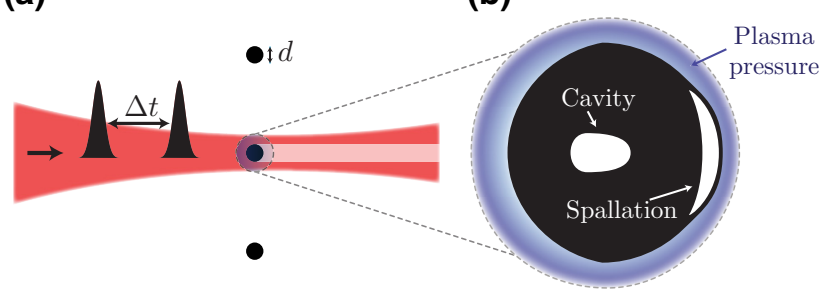

FIG. 1. (a) Illustration of the two laser pulses spaced by a time delay $\Delta t$ incident on a tin microdroplet with diameter $d$. (b) Illustration of the central cavity and spall formation after the first laser impact and the plasma pressure surrounding the droplet as a result of the second pulse impact.

expansion velocity of the main central shell [17]. These fragments can possibly escape interaction with the main pulse, adding to debris, or alternatively, any EUV light generated from this extended part of the target will be lost if it exceeds the etendue of the collection optics. The ability to generate expanded targets without fast spallation debris [25] could therefore be an advantage favoring application of shock-wave-induced deformation.

In this work, we study tin microdroplet deformation induced by impact of two laser pulses having durations of $0.4 \mathrm{~ns}$ delayed by several to tens of nanoseconds. Impact of a single pulse with a duration of 0.4 ns predominantly leads to shock-wave-dominated deformation, comprising central cavity and rear-side spall formation as illustrated in Fig. 1(b). In the following, we demonstrate how the addition of a second pulse alters the observed deformation by excitation of a second shock wave and added plasma recoil pressure applied by an expanding plasma. We discuss the increased complexity in observed deformation, and as a main observation find that the spall-front velocity (from hereon referred to as simply the spall velocity) displays a sharp reduction as a function of interpulse spacing. We attribute this reduction to the backpressure impulse, which is produced by a plasma flow around the droplet that is driven by the second pulse, and which slows down the thin spall layer after its detachment from the main droplet body [see Fig. 1(b)]. We perform radiation-hydrodynamics simulations to gain a better understanding of the proposed spall-velocity-reduction mechanism and have investigated the influence of the energy of the second pulse.

\section{METHODS}

In the experiment, a stream of liquid tin is expelled from a nozzle mounted vertically on top of a vacuum chamber $\left(\simeq 10^{-7}\right.$ mbar $)$. A modulation applied to the nozzle leads to a controlled breakup of the tin stream into equalsized droplets traveling downward at approximately 10 $\mathrm{m} / \mathrm{s}$. By varying the frequency of the modulation we tune the droplet diameter $d$ from 19 to $52 \mu \mathrm{m}$. The droplets then pass a light sheet prepared above the center of the vacuum vessel and scatter some of the light. This scattered light is detected by a photomultiplier tube and is used to trigger the drive laser and data acquisition at a $10 \mathrm{~Hz}$ repetition rate.

Two temporally Gaussian-shaped laser pulses with a full width at half maximum (FWHM) duration of $0.4 \mathrm{~ns}$, wavelength $\lambda=1064 \mathrm{~nm}$, and energies $E_{1}$ and $E_{2}$ of $20 \mathrm{~mJ}$ per pulse $\left(E_{1}=E_{2}\right)$ are incident on the droplet as illustrated in Fig. 1(a). The pulse pair generation is automated using the arbitrary pulse shaping capability of the drive laser [26]. Combined with a straightforward feedback algorithm it allows for a convenient, accurate, and fully electronic tuning of the pulse amplitudes and spacing. The spacing between the two pulses $\Delta t$ is scanned up to $100 \mathrm{~ns}$ with a minimum possible step size of $0.4 \mathrm{~ns}$. The laser beam, with a circular polarization, is focused to a Gaussian spot of approximately $125 \mu \mathrm{m} \mathrm{FWHM}$ at the position of the droplet.

The droplet is backlit using an incoherent laser pulse with $\lambda \approx 560 \mathrm{~nm}$ and the shadow created by the droplet is imaged onto a camera using a long distance microscope. The spatial and temporal resolutions of this shadowgraphy setup positioned perpendicularly to the laser propagation axis are approximately $5 \mu \mathrm{m}$ and $5 \mathrm{~ns}$, respectively. A recording of the droplet deformation is obtained by repeating the experiment while scanning the back-lighting laser pulse in time with respect to the drive laser in steps of $50 \mathrm{~ns}$.

\section{RESULTS AND INTERPRETATION}

An extensive overview of the observed deformation is given in Fig. 2. For each droplet diameter $d$ (ranging from 19 to $52 \mu \mathrm{m}$ ), a selection of $\Delta t$ is made to best illustrate the trends in deformation in the relevant range. First, looking at the single-pulse images for increasing $d$ (Fig. 2, top row), the most notable effect is a change in the ratio between the size of the main cavitation bubble and the spall. For this shock-wave deformation regime, an asymptotic hydrodynamic similarity (of the internal pressure evolution) is shown to exist when the total deposited energy $\left(Q_{\text {tot }}\right)$ per droplet volume $Q_{\text {tot }} / d^{3}$ is kept constant [17]. This similarity is supported by the observed collapse of the radial expansion velocities of the main central cavitation bubble presented in Refs. [18,19]. Since, in this work, the laser focal spot size and laser energy are kept constant throughout the experiment, both the energy impinging on the droplet $E_{\text {od }}$ as well as the droplet volume vary significantly when changing $d$. Therefore, assuming that $Q_{\mathrm{tot}} \propto E_{\mathrm{od}} \propto d^{2}$, we find that the similarity noted in Ref. [17] is not obeyed in our case. Since $E_{\mathrm{od}} / d^{3} \propto 1 / d$, we see in Fig. 2 a decrease in the size of the central cavitation bubble and spall with increasing $d$. 

(a) $d=19 \mu \mathrm{m}$
(b) $d=26 \mu \mathrm{m}+$
(c) $d=36 \mu \mathrm{m}$
(d) $d=44 \mu \mathrm{m}$
(e) $d=52 \mu \mathrm{m}$
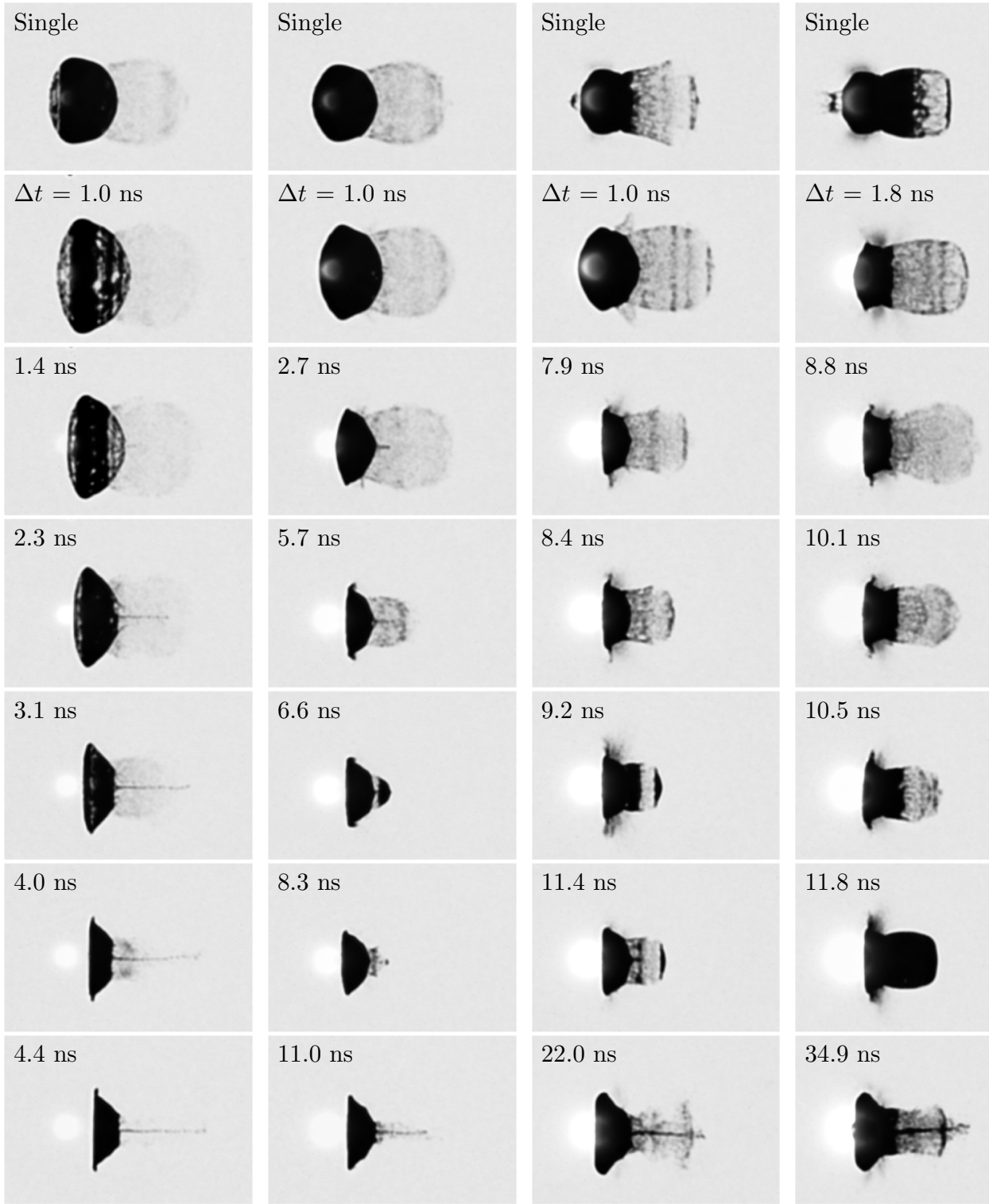
$8.4 \mathrm{~ns}$
10.1 ns
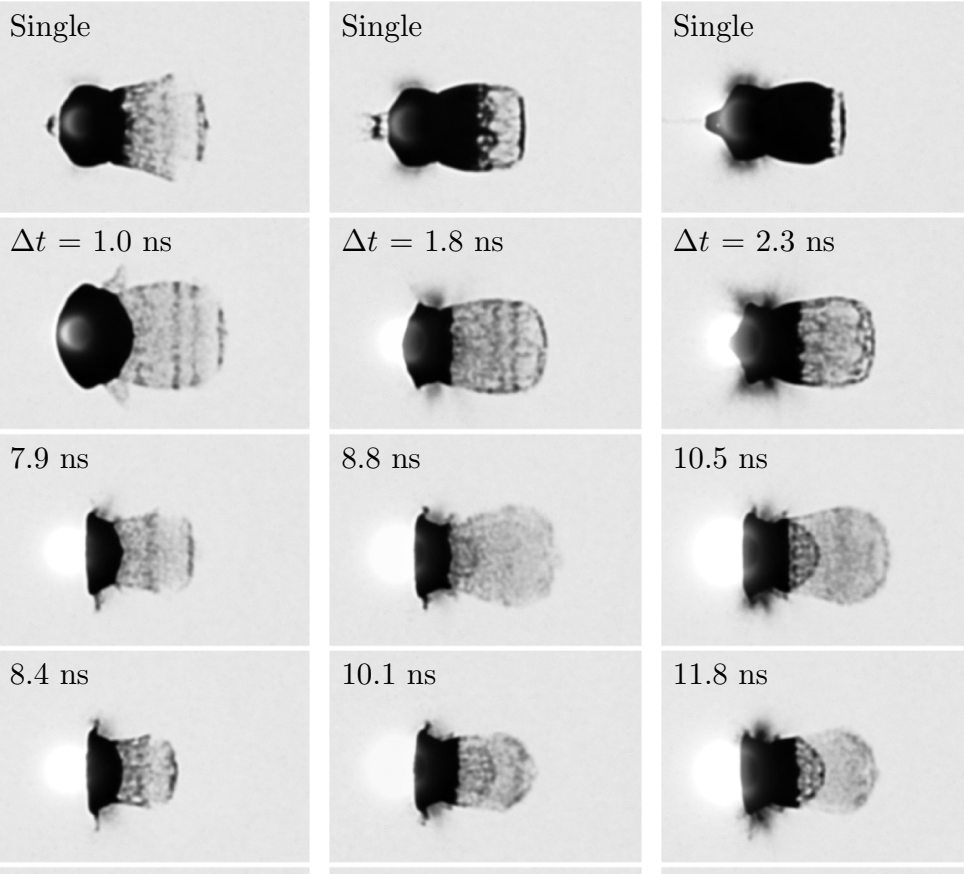
$9.2 \mathrm{~ns}$
$10.5 \mathrm{~ns}$
$10.5 \mathrm{~ns}$
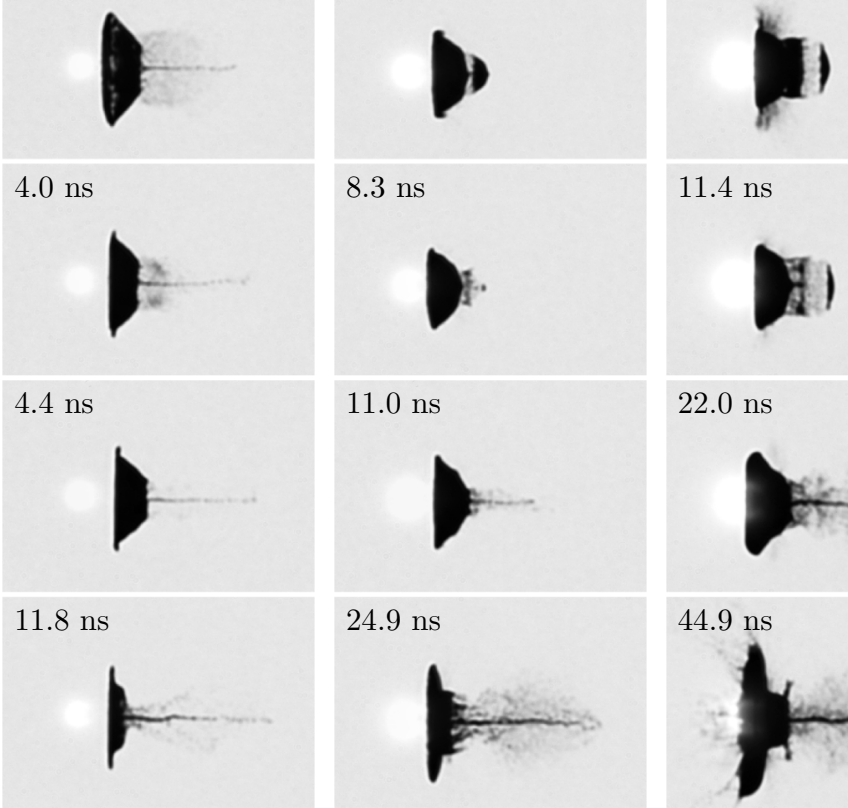
$11.4 \mathrm{~ns}$
$11.8 \mathrm{~ns}$
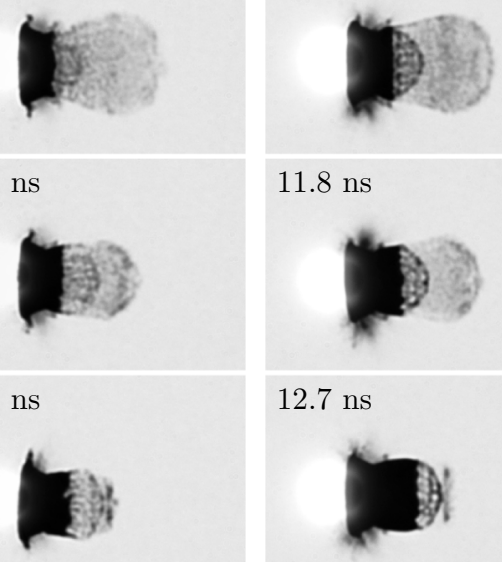
$11.8 \mathrm{~ns}$
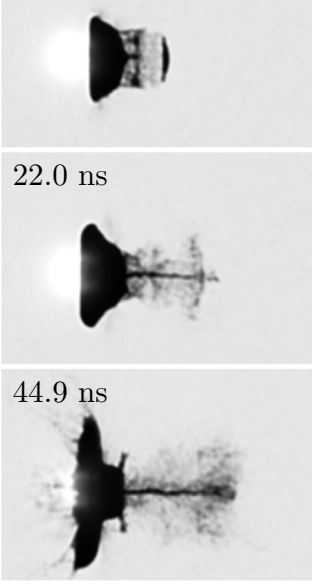
$50.1 \mathrm{~ns}$
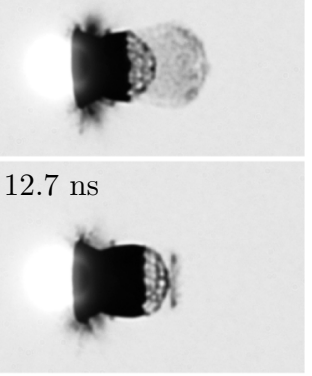
$t=0.4 \mu \mathrm{s}$

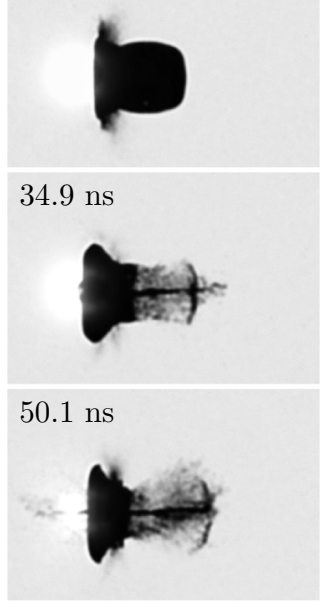
$13.6 \mathrm{~ns}$
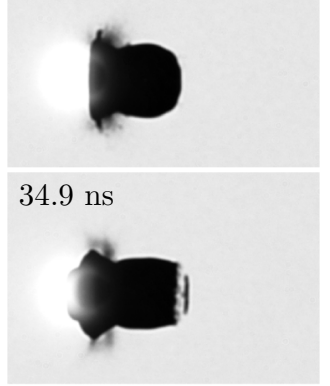
$60.1 \mathrm{~ns}$

FIG. 2. Side-view shadowgram overview of the observed deformation. Two laser pulses, each with a duration of $0.4 \mathrm{~ns}$, and energy of $20 \mathrm{~mJ}$, are incident from the left, focused down to a Gaussian spot with a full width at half maximum of $125 \mu \mathrm{m}$, and spaced by a time delay $\Delta t$. All shadowgrams are recorded $t=0.4 \mu \mathrm{s}$ after impact of the first laser pulse. From left to right, columns (a)-(e) show the different droplet sizes (with diameter $d$ ). The rows show increasing values of $\Delta t$, independently chosen per droplet size to best illustrate the observed trends in deformation. The bright spot is plasma emission, which, although it has a duration similar to the laser pulse (approximately nanoseconds), is visible at $t=0.4 \mu \mathrm{s}$ due to the long exposure time of the CCD camera (approximately milliseconds).

With the introduction of a second laser pulse we observe several changes in the target morphology that strongly depend on $\Delta t$. First, we see a flattening of the front, laser facing surface due to the second pressure kick. An increase in the size of the visible plasma cloud suggests that this pressure is partly supplied by reheating of the 
plasma initially generated by the first pulse [25]. Remarkably, effects of the second pressure kick on the transverse (vertical) size of the main expanding body, and hence the expansion velocity, are minor. In other words, although with the addition of a second pulse extensive flattening is present leading to a considerably different shape, the radial size is similar to the single pulse case [see, for example, Fig. 2(a), $\Delta t=11.8 \mathrm{~ns}$ ]. Second, a strong jetting behavior is observed, most clearly visible in Fig. 2(a). The origin of the microjets and the observed trends are qualitatively discussed in Sec. III D. Finally, clear changes in the spall dynamics are apparent. From Fig. 2, it is already clear that, for all droplet sizes, an initial increase in the spall velocity with increasing $\Delta t$ is followed by its strong decrease at still larger $\Delta t$. Furthermore, for the largest two droplet sizes, we observe a second spall-like front following the initial spall. The increased droplet size reduces $Q_{\text {tot }} / d^{3}$ and results in a reduced amplitude of the shock wave and the trailing rarefaction wave. This in turn means that the region where the maximum negative pressure exceeds the tensile strength of the liquid is reduced, and therefore the region of vapor void formation, i.e., the central cavity, will be smaller and expands at a reduced rate. A relatively small cavity size at early times and its reduced expansion rate can allow for a second shock wave to pass, enabling it to generate the second spall front in a similar manner to the generation of the first. The observation of this second spall event is considered evidence of the existence of a second shock wave launched by the impact event of the second pulse. A larger cavity size, which is attained at large $\Delta t$, or by decreasing $d$ (and thus increasing $Q_{\text {tot }} / d^{3}$ ), likely blocks passage of the second shock wave and prevents a second spall from being formed. Indeed, for the smaller droplet sizes, the second spall is absent.

At small $\Delta t$ the close proximity of the two shock waves can lead to more complicated dynamics and we presume that an exceedingly complex pressure wave evolution is likely hidden inside the droplet interior. Even at $\Delta t=1 \mathrm{~ns}$ we find that the resulting deformation is profoundly different from the case of a single pulse with a combined energy. We can identify several deformation features that suggest a joint influence of both shock waves on the central cavitation and spall formation. These include the increased radial extent of the central cavity clearly visible in Fig. 2(a) at $\Delta t=1.0$ and 1.4 ns and in Figs. 2(b) and 2(c) at $\Delta t=1.0 \mathrm{~ns}$. Further indications are the notable changes in spall morphology and an increase of the spall velocity for all $d$ at the lowest values of $\Delta t$ (represented by the second row in Fig. 2). As will be shown later, the impact of the second pulse leads to a significant plasma pressure even at the back side of the droplet. This will generate counterpropagating shock waves in the droplet that may interfere with the shock wave initiated by the first pulse, thereby complicating the internal pressure distributions and the spall formation further. A full explanation of all observations presented in Fig. 2 is beyond the scope of the current work and would require complex dedicated simulations incorporating both accurate fluid and plasma modeling with improved knowledge of the equation of state and spall strength of liquid tin [16,27,28].

\section{A. The spall velocity}

By tracking the spall front throughout the side-view shadowgraphy image sequence we obtain its trajectory. At the laser energies used we find the spall front trajectories (before and after fragmentation) to be fully ballistic and not significantly hampered by surface tension. The spall velocity, which we denote $u_{s}$, is therefore straightforwardly obtained using a linear fit and a presentation of the spall front position as a function of the shadowgraphy time delay is omitted. The obtained $u_{s}$ for different droplet diameters as a function of the interpulse delay $\Delta t$ is shown in Fig. 3.

The obtained spall velocities for single pulses range from approximately 300 to $600 \mathrm{~m} / \mathrm{s}$ and can be used to estimate the peak pressure of the shock wave when it arrives at the back side of the droplet, i.e., during spall formation. This is done using the velocity doubling rule, which states that $u_{f}=2 u_{m}$ [29], where $u_{f}$ is the velocity of the free surface (the droplet surface) and $u_{m}$ is the material velocity (for a brief derivation, see also the supplementary information of Ref. [30]). The material velocity $u_{m}$ is related to the pressure $P$ by $P=u_{m} \rho_{0} c_{s}[29,30]$, with $\rho_{0}=7 \times 10^{3} \mathrm{~kg} \mathrm{~m}^{-3}$ the density of liquid tin [31] and $c_{s}$ the shock velocity. When approximating $c_{s}$ by the speed of sound in liquid tin $\left(2.5 \mathrm{~kg} \mathrm{~m}^{-1}\right.$ [32]), we find that the pressure of the wave when reaching the back side of the droplet ranges from approximately 2 to $5 \mathrm{GPa}$ in our experiment. Similar peak pressures at the back side of the droplet and more detailed analyses of the shock-wave pressure evolution through its propagation have been reported in Refs. [15-17].

In the above estimate of the shock pressure we have to consider that, due to our limited temporal and spatial resolution, we are unable to resolve the early time $(<10 \mathrm{~ns})$ dynamics of the free surface $[20,30,33]$ that, in a rather simplified picture, result from reflections between the spall plane (the ruptured region) and said free surface. The spall "release" velocity $u_{s}$ as measured is lower than the relevant free surface velocity $u_{f}$ at first arrival of the shock wave, but approaches $u_{f}$ for higher $u_{s}$ [30]. For this reason, an estimate of $P$ obtained from the spall velocity $u_{s}$ might be more accurate for the larger velocities. Nevertheless, following the above, and since $c_{s}>c$, the deduced pressure should be considered a lower bound estimate.

\section{The effects of interpulse spacing $\Delta t$}

For all droplet sizes, we can identify a clear, rather sudden reduction in $u_{s}$ when increasing $\Delta t$, as shown in Fig. 3. This reduction shifts to earlier times with decreasing $d$. 


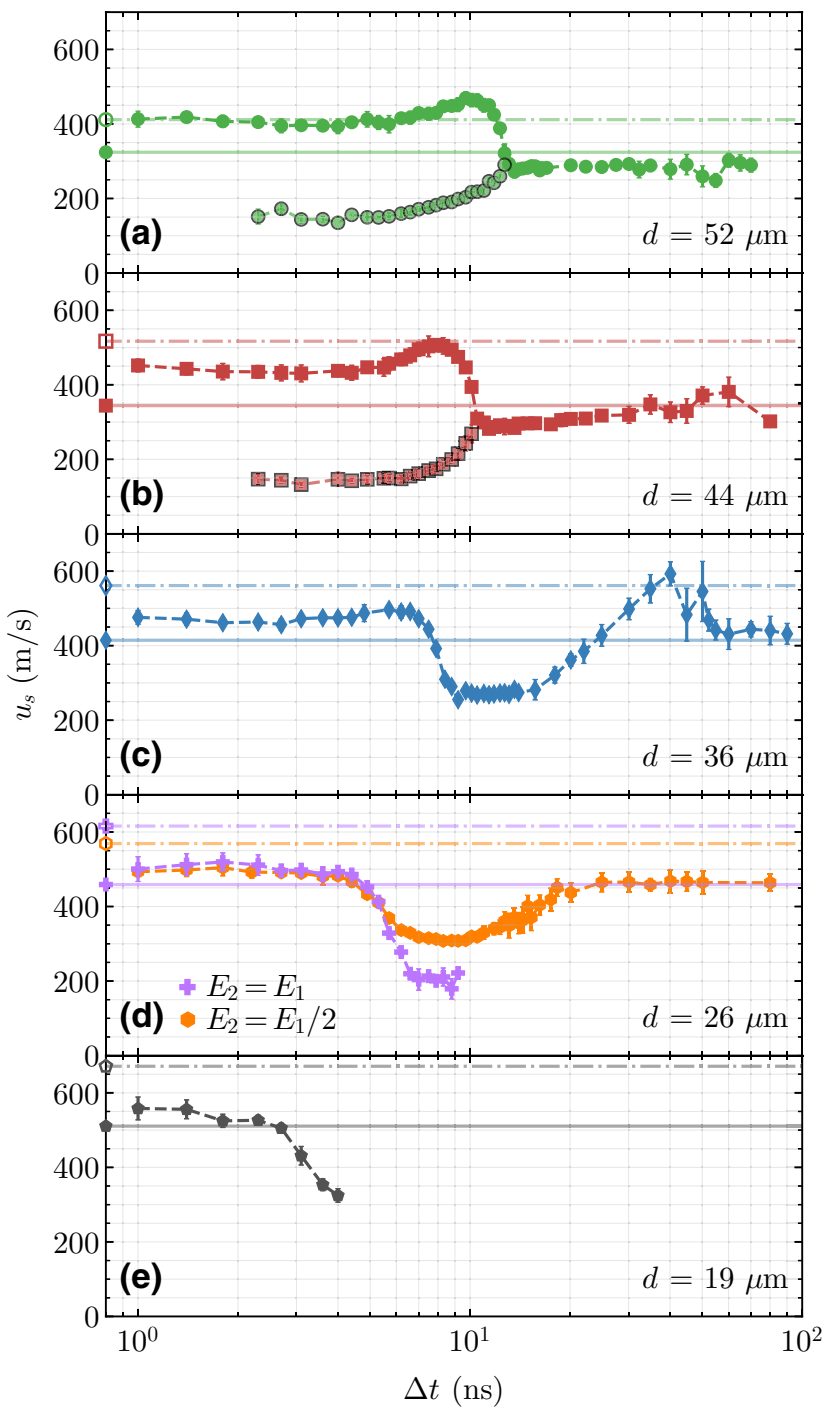

FIG. 3. Spall velocity $u_{s}$ for all droplet sizes as a function of the interpulse delay $\Delta t$. The solid horizontal line corresponds to the spall velocity observed following impact of a single pulse with an energy equal to only the first pulse $\left(E_{1}\right)$. The dash-dot line corresponds to the spall velocity after impact of a single pulse with the combined energy $\left(E_{1}+E_{2}\right)$. The markers with a black outline in (a) and (b) are the velocities of the second (inner) spall.

The behavior of the spall velocity before and after this reduction differs slightly for different droplet sizes. For $d=$ $19 \mu \mathrm{m}$, a reduction in $u_{s}$ is observed after a short plateau. Because of the strong jetting behavior, the spall velocity cannot be tracked beyond $\Delta t=4 \mathrm{~ns}$. For $d=26 \mu \mathrm{m}$, we observe a similar general trend, but since the jet velocity is reduced, we can track $u_{s}$ until a plateau is reached, completing a transition before tracking of the spall is impeded. For $d=36,44$, and $52 \mu \mathrm{m}$, the reduction is preceded by a minor increase, and after the reduction a recovery to the original single-pulse spall velocity is present. For $d=36 \mu \mathrm{m}$, the recovery happens after an increase, possibly due to the propulsion by a plasma cloud reaching the spalled shell from behind. From Fig. 2, it is also clear that at these relatively large $\Delta t$ the original spall front is already disrupted and the tracking accuracy is compromised. Finally, for $d=44$ and $52 \mu \mathrm{m}$, we observe a second spall front whose velocity is indicated by the dark edged markers in Fig. 3. The velocity of this inner spall starts low and increases towards the transition point at which it "merges" with the outer spall. The second spall is caused by the second shock wave, as generated by the second laser pulse, which traverses through a complex medium, i.e., through a state of matter resulting from the rarefaction following the first shock (in the single pulse case a central vapor cavity is formed, for example). A full description of shock propagation through such, yet poorly understood and complex, medium is beyond the scope of the current manuscript, and the exact cause of the acceleration of the second spall with increasing $\Delta t$ remains unclear.

To check the sensitivity of the observed transition time to $E_{2}$, an extra measurement is performed for $d=26 \mu \mathrm{m}$ in which $E_{2}=E_{1} / 2$ instead of $E_{2}=E_{1}$ [see Fig. 3(d)]. Comparing the two cases we see that the moment of transition shows no significant shift. Furthermore, reducing $E_{2}$ leads to a less-prominent reduction in $u_{s}$ (down to $300 \mathrm{~m} / \mathrm{s}$ instead of $200 \mathrm{~m} / \mathrm{s}$ ), and to a less prominent jetting behavior. As a result of the lower jet velocities, we can fully track the recovery of $u_{s}$ to the single pulse value.

\section{B. Mechanism of the spall-velocity reduction}

\section{On a possibility of destructive interference of two successive shocks}

Now we turn to a possible physical mechanism that could explain the observed reduction of $u_{s}$, caused by a properly delayed second laser pulse. Here, one of the key factors is the position of the spall "plane," i.e., of the surface where the liquid under tensile strain is ruptured. Although the exact location of this surface is, strictly speaking, unknown in our experiment and changes with $d$, we have, on the basis of the available simulation work $[16,17]$, all grounds to believe that it lies at a typical depth of approximately $d / 20$, i.e., relatively close to the rear surface of the droplet. This is also corroborated by the observed early fragmentation of the spall layer during its subsequent expansion $[18,19]$.

A tempting idea would be to try to explain the $u_{s}$ reduction by the fact that a properly timed second shock, launched by the second laser pulse, catches up with the first shock just in time to cancel (or to reduce in amplitude) the negative-stress (tensile) wave behind the latter, thus preventing the spallation event. In this picture, however, the shallow depth of the spall plane, combined with the relevant times observed in the experiment, would imply unrealistically high velocities of the second shock needed 
to catch up with the first one and interfere with the reflected tensile wave. Indeed, for the 52- $\mu \mathrm{m}$-diameter droplet, the observed decrease of $u_{s}$ happens at $\Delta t \approx 12 \mathrm{~ns}$. If we take conservative values and assume a spall fracture plane depth of $d / 10$ and approximate the first shock-wave velocity with the speed of sound $c$ of $2500 \mathrm{~m} \mathrm{~s}^{-1}$, then for the second shock wave to reach the spall plane before or at the moment of spall inception, its velocity would have to be $\geq 1.7 c$. If we take a propagation velocity of the first shock wave of $1.2 c$ then the velocity of the second shock wave would need to be $\geq 2.7 c$. Such differences in velocity between the two shock waves cannot readily be explained.

Second, for the 36- and 26- $\mu$ m-diameter droplets, the reduced spall velocity remains low for approximately 10 ns after its initial drop. If the wave interference were the cause, we would expect this phase to not last longer than any possible overlap of the waves. An estimate of the width of the reflected tensile wave can be obtained from the simulation in Ref. [17], from which we infer it to be no larger than $d / 5$. This is also confirmed by the above observation that the width of the spalled layer is of the order of $d / 20$. For $d=36 \mu \mathrm{m}$, the duration of overlap of two counterpropagating waves with such a width, again approximating their propagation velocity by $c$, would last only $0.2 \times 36 \mu \mathrm{m} / 2 c \approx 1.4 \mathrm{~ns}$, an order of magnitude below the observed prolonged spall velocity suppression.

Thus, having effectively ruled out the wave interference as the main cause of the spall-velocity reduction, we next demonstrate that this reduction can in fact be explained by the counterpressure of the ejected plasma cloud that, having been accelerated by the second laser pulse, flows round the droplet and converges upon the symmetry axis in front of the backward-moving shell of the spalled material.

\section{Simulation of the plasma pressure evolution.}

To gain insight into the mechanism of the observed spall-velocity reduction, we have performed radiationhydrodynamics simulations using the RALEF-2D code [34-36]. In recent years, this code has been employed in numerous EUV source-plasma related topics, ranging from droplet propulsion studies [6] to plasma modeling $[3,37,38]$. In our simulations, the pressure evolution within the (neutral) liquid tin droplet and the resulting deformation strongly depend on the choice of the equation-of-state model [16], and have therefore not been investigated. Instead, our main purpose is to examine the plasma dynamics resulting from the impact of the second laser pulse.

The simulations have been performed with pulse energies and an illumination geometry equivalent to the experiments for two configurations: $d=19 \mu \mathrm{m}$ (with $\Delta t=8 \mathrm{~ns}$ ) and $d=52 \mu \mathrm{m}$ (with $\Delta t=12 \mathrm{~ns}$ ). The results, presented in Fig. 4, show that after impact of the second pulse the expanding plasma "wraps" around the droplet and a significant plasma pressure develops at the back side of the

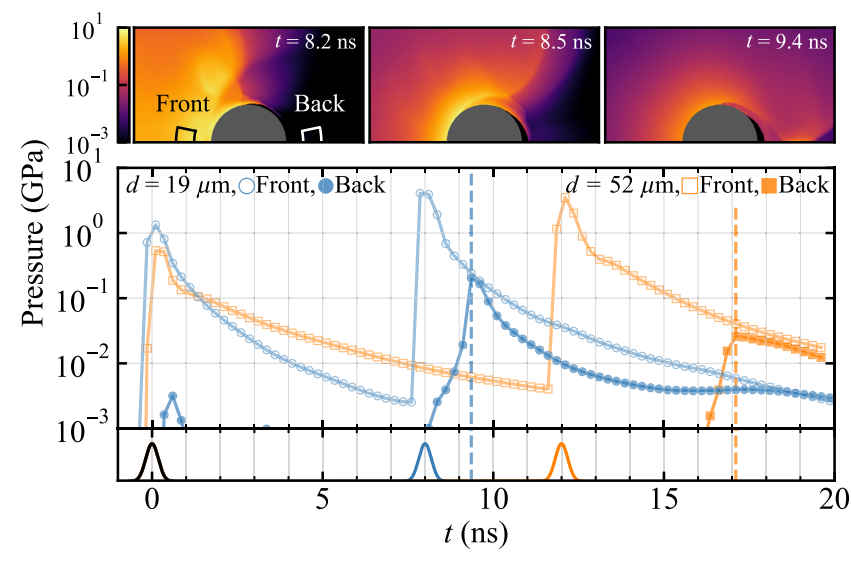

FIG. 4. Plasma pressure evolution during double-pulse illumination as simulated using the RALEF-2D radiationhydrodynamics code for $d=19$ and $52 \mu \mathrm{m}$. The top hemisphere of the droplet $(d=19 \mu \mathrm{m})$ is shown (top) for three selected time frames to illustrate the "wrapping around" of the plasma pressure after impact of the second pulse. Two regions of interest, one at the front and one at the back of the droplet, are chosen. The average pressure inside both regions is plotted as a function of time $t$ (bottom). The corresponding laser pulse sequences are shown in the attached bottom most panel, with the first pulse (black) common to both droplet sizes. For $d=19 \mu \mathrm{m}$, the second pulse is incident at $t=8 \mathrm{~ns}$ (blue), and for $d=52 \mu \mathrm{m}$, the second pulse is incident at $t=12 \mathrm{~ns}$ (orange).

droplet (where the spall forms). It is this pressure that we attribute the reduction in $u_{s}$ to when it coincides with the moment of spall formation, i.e., when the shock wave from the first pulse reaches the back side of the droplet. The plasma flow around the droplet, which results in the accumulation of a relatively dense and hot cloud on the laser axis, has previously been reported on for the case of single $10 \mathrm{~ns}$ pulses and was found to exert a noticeable backward pressure [6].

Knowing the magnitude $P$ and the effective duration $\tau$ of the rear-side plasma pressure, we can apply the condition of momentum balance $P \tau \sim \rho b v$ to verify that the pressure impulse $P \tau$ would indeed be sufficient to reduce the velocity of a spall layer of thickness $b$ and density $\rho$ by a value $v$. The magnitude of the pressure at the back side of the droplet peaks at approximately $0.2 \mathrm{GPa}$ for the $19 \mu \mathrm{m}$ droplet example case. The observed spallvelocity reduction of $300 \mathrm{~m} / \mathrm{s}$ due to a pressure impulse of $P \tau \sim 0.2 \mathrm{GPa} \times 0.4 \mathrm{~ns}$ implies (taking $\rho=\rho_{0}$ as an upper limit) a layer thickness $b \approx 40 \mathrm{~nm}$. For the $52 \mu \mathrm{m}$ droplet, the peak pressure at the back side of the droplet is approximately $0.02 \mathrm{GPa}$, an order of magnitude lower than for the $19 \mu \mathrm{m}$ case. However, since this pressure persists longer, namely for a minimum of around $3 \mathrm{~ns}$ (at which our simulation ends), and the spall-velocity reduction is less (approximately $100 \mathrm{~m} / \mathrm{s}$ ), the same momentum balance implies a layer thickness $b \approx 90 \mathrm{~nm}$. These estimated spall layer thicknesses are in line with expectations from the 
aforementioned detailed simulation efforts [16,17] on the one hand, and several complementary experimental efforts on the other. Experiments by Krivokorytov et al. [15] found a partial transparency of a late-time sheet that would imply a 10-100 nm thickness; experiments by Kurilovich et al. [18] uncovered a hydrodynamic scaling relation of hole formation in an expanding thin layer that yields a rough $\sim 200 \mathrm{~nm}$ thickness estimate when applied to our observations. These theoretical and experimental estimates of the spall layer thickness strongly support the argument of a plasma pressure impulse slowing down the detached front spall when its arrival on the back side of the droplet is timed to coincide with the detachment of the first spall.

The rapid decay of the plasma pressure (see Fig. 4) implies that if the second pulse is "early," i.e., it impacts before the shock wave from the first pulse reaches the back side of the droplet, no slowing down of the spall should be present. If the second pulse is "late," i.e., it impacts at a later moment at which the spall has already expanded significantly, the plasma is forced to expand over a larger distance and will decrease in density. This is likely to decrease the recoil pressure it exerts on the spall and allow for a recovery of $u_{s}$. Both arguments are in agreement with our observations.

Crucially, this knowledge allows us to relate the moment of spall release to the impact time of the second pulse by the observed reduction of $u_{s}$, which will be further detailed in the following section. Evidently, we need to take into account the delay between the moment of pulse impact and the pressure peak. From the simulations we observe an approximate delay of 1.4 and $5.1 \mathrm{~ns}$ for $d=19$ and $52 \mu \mathrm{m}$, respectively, and linearly interpolate this plasma expansion delay with $d$.

\section{The shock-wave velocity}

The instantaneous velocity of the first shock varies as it propagates from the front to the back side of the droplet and heats up and sets in motion an ever increasing fluid mass. On average, the shock becomes weaker, although a certain local and transient amplification may be observed when it converges towards the droplet center [17]. Although in our experiment we do not have access to the instantaneous velocity, we can determine an average shock-wave speed from the spall release moment, assuming that the spall formation time is small compared to the total propagation time of the shock wave.

To obtain the spall release moments $t_{s}$, we first determine the half-way position of the transition, i.e., the reduction in $u_{s}$, and its width by locally fitting an error function to the data shown in Fig. 3. The results of these fits are indicated in Fig. 5(a) by open markers. For $d=19 \mu \mathrm{m}$, where the transition is not complete, a broad estimated range for the value of the plateau after the reduction is taken from 100 to $300 \mathrm{~m} / \mathrm{s}$ for the fitting. The corresponding spread
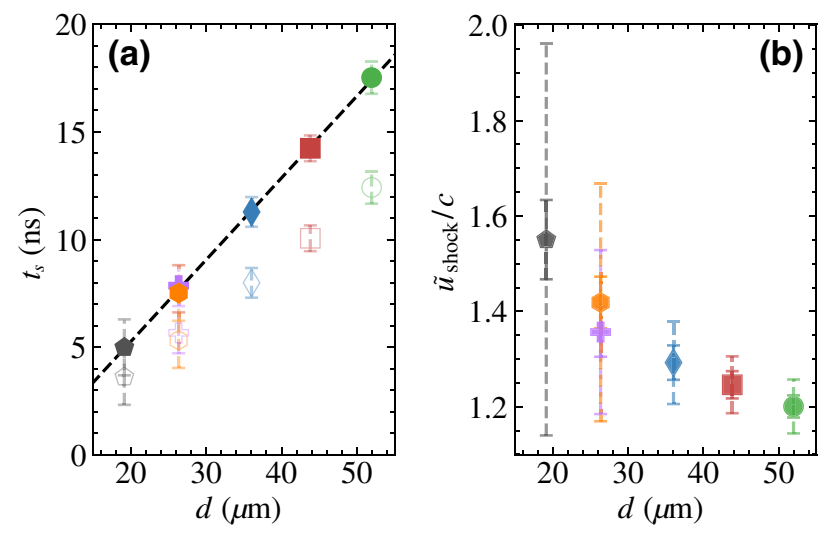

FIG. 5. (a) Spall release moment $t_{s}$ (filled markers) as a function of droplet size $d$. Open markers correspond to the moment of reduction of $u_{s}$, as determined by an error-function fit to the data presented in Fig. 3, without the added plasma-delay correction. Error bars indicate the $1 \sigma$ width of the error function fit and are used as weights in the presented fit of a linear curve. The fit parameter standard errors of $t_{s}$ are smaller than the markers. (b) The average shock-wave velocity $\tilde{u}_{\text {shock }}=d / t_{s}$ normalized to the speed of sound $c$. The outer (dashed) error bars correspond to the propagated error shown in (a). The inner (solid) error bars correspond to the propagated fit standard error. The error in droplet diameter is estimated to be $\pm 1 \mu \mathrm{m}$.

in the obtained value of $t_{s}$ is reflected in the error bar. The true spall release moment $t_{s}$ is subsequently determined by adding the previously mentioned plasma peak delay with respect to the second laser pulse and is plotted in Fig. 5(a) using filled markers. We find a linear dependence of $t_{s}$, and by a least-squares fit to the data obtain a slope of $0.38(1) \mathrm{ns} / \mu \mathrm{m}$ and an intercept of $-2.3(2) \mathrm{ns}$.

The linear dependence $t_{s} \propto d$ suggests a single common average shock-wave velocity for all $d$. However, because $E_{\text {od }} / d^{3}$ varies with droplet size, the aforementioned hydrodynamic similarity [17] does not hold and we expect a dependency of the average shock-wave velocity on $d$. The observed offset of the fit is also not in agreement with a single velocity, since in that case we do not expect an offset, i.e., $\lim _{d \rightarrow 0}\left(t_{s} \rightarrow 0\right)$. We therefore rationalize that the obtained slope should approach the asymptotic velocity of the pressure wave, i.e., the speed of sound $c$, and obtain a velocity $u_{a}=1 / 0.38(1)=2.63(4) \mathrm{km} / \mathrm{s}$, which, taking $c=2.47 \mathrm{~km} / \mathrm{s}$ [32], indeed corresponds to $u_{a} / c=$ $1.07(2)$, being close to unity.

Since we expect a different shock velocity for each droplet size, it is sensible to treat them independently and obtain an average shock velocity $\tilde{u}_{\text {shock }}=d / t_{s}$ for each droplet size, as shown in Fig. 5(b). The negative $t_{s}$ offset in Fig. 5(a) entails an increasing $\tilde{u}_{\text {shock }}$ with decreasing $d$ as expected by the increasing $Q_{\text {tot }} / d^{3}$. The obtained values for $\tilde{u}_{\text {shock }}$, ranging approximately from $1.6 c$ to $1.2 c$ between the smallest and largest droplet, respectively, are comparable to the $1.25 c$ we deduce from Ref. [17]. In Fig. 5(b) two 
error bars are plotted for each data point. The inner (solid) error bar corresponds to the propagated combined standard error of the relevant fit parameter and the estimated error in the droplet size of $\pm 1 \mu \mathrm{m}$. In Fig. 5(a), this error is smaller than the marker size. For the larger (dashed) error bars, the $1 \sigma$ width of the error function fit representing the transition width [which is the error bar shown in Fig. 5(a)] is included. The inclusion of this error displays the sensitivity to the choice of $t_{s}$ that we set here at the half-way $(50 \%)$ point. Figure $5(\mathrm{~b})$ shows that the accuracy with which $\tilde{u}_{\text {shock }}$ can be retrieved using this method is inherently limited for smaller droplet sizes without additional assumptions or modeling to motivate a better choice of $t_{s}$ along the $u_{s}$ curve. Also, for small $d$, the one-way propagation time of the shock wave (given by $t_{s}$ ) approaches the pulse duration $(0.4 \mathrm{~ns})$ and the hydrodynamic timescale of the plasma expansion, and possibly also the spall formation duration. All in all, the error in $\tilde{u}_{\text {shock }}$ for the 44 and $52 \mu \mathrm{m}$ droplets is nonetheless below $10 \%$.

\section{The effects of the second pulse energy}

For the case $d=26 \mu \mathrm{m}$, we perform two measurement series in which $E_{2}$ is varied at a fixed $\Delta t$. One $\Delta t$ value is chosen on either side of the spall-velocity transition, specifically at 3.6 and $7.6 \mathrm{~ns}$. The results are shown in Fig. 6. At $\Delta t=3.6 \mathrm{~ns}$, before the transition, the spall velocity (top) undergoes a gradual minor increase. Inversely, in agreement with the proposed spall-velocity reduction mechanism, $u_{s}$ displays a strong decrease with increasing $E_{2}$ at $\Delta t=7.9 \mathrm{~ns}$.

When increasing $E_{2}$ beyond $E_{1}$, we observe a strong difference in the transverse expansion velocities, quantified at early times and plotted in Fig. 6(a)(bottom). Up to
$E_{2}=E_{1}$ the initial expansion velocity $\dot{R}(t=0)$ is rather flat and equal for both values of $\Delta t$. When $E_{2}>E_{1}$, a separation of the two cases takes place, most notably driven by an increase of expansion when $\Delta t=3.6 \mathrm{~ns}$. From the accompanying shadowgrams [see Fig. 6(b)], it appears that this increased expansion is driven by additional cavitation induced by the second pulse.

Although a full picture of the pressure evolution in the droplet interior is likely needed to reveal the origin of this increased expansion, we speculate that, when $E_{2}>E_{1}$, the shock wave induced by the second pulse can be larger in amplitude than the first and will lead to cavitation at a slightly earlier point during its converging trajectory towards the droplet center. Additionally, at $\Delta t=3.6 \mathrm{~ns}$ the cavity induced by the first pulse is either very small or still in the formation phase [17,30]. It is therefore possible that two cavitation regions are formed independently that merge at an early time during their expansion. In line with this reasoning we see that no jet is formed in this case. Furthermore, since no strong increase in $u_{s}$ is present, we have gained access to a target shape [see Fig. 6(b) $(*)$ ] distinct from any single pulse case in its ratio between radial expansion and spall velocities. This target demonstrates the potential of the double-pulse approach to fine tune the target formation process with additional control.

Compared to $\Delta t=3.6 \mathrm{~ns}$, with $\Delta t=7.9 \mathrm{~ns}$, no increase in the initial expansion velocity due to additional cavitation (spherical expansion) is observed when $E_{2}>E_{1}$. Instead, increasing $E_{2}$ leads to increased flattening and jetting. An advanced growth of the cavity from the first pulse at $\Delta t=$ $7.9 \mathrm{~ns}$ compared to $\Delta t=3.6 \mathrm{~ns}$ will impede any additional cavitation by the second pulse as the shock wave will simply impact the cavity. This again allows for the formation (a)

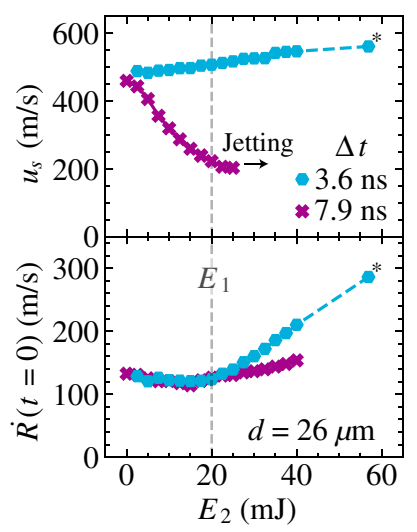

(b) $t=0.4 \mu \mathrm{s}$

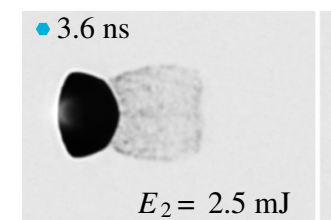

* $7.9 \mathrm{~ns}$

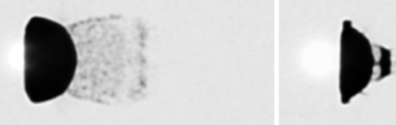

$E_{2}=2.5 \mathrm{~mJ}$

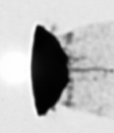

$22.5 \mathrm{~mJ}$

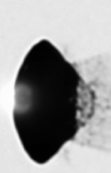

$37.5 \mathrm{~mJ}$
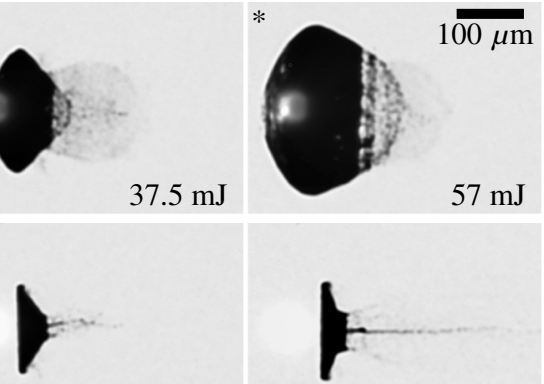

$17.5 \mathrm{~mJ}$

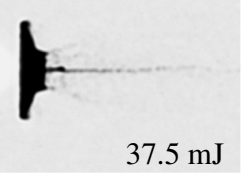

FIG. 6. (a) Effect of $E_{2}$ on the spall velocity $u_{s}$ and initial expansion velocity $\dot{R}(t=0)$ for interpulse delay $(\Delta t)$ values of 3.6 and $7.9 \mathrm{~ns}$. (b) Shadowgrams accompanying data in (a), with the top and bottom rows showing images for $\Delta t=3.6 \mathrm{~ns}$ and $\Delta t=7.9 \mathrm{~ns}$, respectively. Columns show increasing $E_{2}$ from left to right with values chosen such that the deformation trend for each case of $\Delta t$ is demonstrated best. The images are recorded $0.4 \mu \mathrm{s}$ after laser impact. Data points and shadowgram of a particular target of interest, as mentioned in the main text, are indicated by the symbol *. 
of a jet and flattening assisted by the additional plasma pressure.

\section{Jetting}

Jet formation is a prevalent phenomenon resulting from the nonspherical collapse of an embedded cavity [39-43]. Besides an already present asymmetry in the cavity surroundings, e.g., a hard surface, or a free surface, a passing pressure field or shock wave can induce a similar type of jetting [44-53]. In the case of laser impact on microdroplets the formation of a jet on the principal symmetry axis (i.e., the laser propagation axis) has been observed in multiple studies $[4,8,25,54,55]$ and has in most cases been attributed to the collapse of an internal cavity. We can say in all likelihood that the jets we observe in Figs. 2 and 6(b) originate from a (partial) collapse of the cavity formed by the first pulse. Here, the shock wave generated by the second pulse is likely responsible for initiating the jetting when it impacts the cavity [54]. The presence of jets in single pulse cases with pulse durations similar to the acoustic timescale $\left(R_{0} / c\right)$ [8] suggests that the pressure field and deformation resulting from impact of longer pulses can function similarly.

Although a detailed quantification of the jetting dynamics is outside the scope of this work, a clear dependence of the jet velocity with pulse spacing $\Delta t$ is present [see Fig. 2(a)]. It has been shown that the velocity of a jet formed by shock-wave impact on a cavity depends on the initial size of the cavity $[44,53,56]$. It is therefore straightforward to conclude that the jet velocity increases with $\Delta t$ since the cavity has more time to grow, resulting in a stronger collapse. The magnitude of the pressure wave inducing the collapse of the cavity and the accompanying jetting is also likely to play a role in the jet velocity as supported by the increase in jet length visible in Fig. 6(b)(7.9 ns). Since the absorbed laser energy fraction may also exhibit a (positive) $\Delta t$ dependence due to the plasma expansion, separating the effects of laser pulse energy and $\Delta t$ on the jet velocity remains challenging.

Accurate tracking of the jet velocity in the current experiments is, in many cases, hampered by the presence of the spall. It either blocks the jet optically, making it impossible to visualize, or it physically blocks the jet in its path when the jet overtakes the spall, causing a disruption of the jet. Therefore, a further study with an in-depth quantification would benefit from different experimental conditions and is left for future work. Considerations for a follow-up study could comprise a lower $E_{1} / d^{3}$ value, below the spallation but above the cavitation threshold $[12,17,18]$, which would prevent spallation from occurring. Another possibility is having a higher $E_{1} / d^{3}$, which would increase the spall velocity and decrease the spall fragmentation time, and thereby improve visibility of the jet. An increased $E_{1} / d^{3}$ can possibly be combined with a lowered $E_{2}$ value to decrease the jet velocity and prevent interaction between the jet and (faster) spall.

\section{CONCLUSIONS}

In conclusion, we have studied the deformation of tin microdroplets after irradiation by a pulse pair with pulses of $0.4 \mathrm{~ns}$ in duration with varying interpulse time delay. As in the single pulse case, we observe the formation of an expanding central cavity and a spall layer, both induced by a shock wave and the accompanying rarefaction wave traversing the droplet. The addition of a second pulse predominantly leads to flattening, jetting, and changes in the spall velocity, all dependent on the interpulse spacing. More specifically, we find a sharp reduction in the spall velocity at a specific droplet-size-dependent pulse spacing. We attribute this reduction to a deceleration of the spall by the plasma pressure resulting from the impact of the second pulse.

Simulations performed with the radiation-hydrodynamics code RALEF-2D confirm that a significant external plasma pressure is able to build up above the rear-side droplet surface undergoing spallation. By combining the results from experiment and simulation we are able to estimate the moment of spall formation, i.e., the arrival time of the shock wave at the back side of the droplet. We find average shock-wave propagation velocities ranging from 1.2 to 1.6 times the speed of sound.

We have discussed the effects of the relative energy of the second pulse at two fixed pulse delays on the various processes involved. We find that, when the energy of the second pulse exceeds the first, the central cavity formation is altered, enhancing the transverse expansion of the final target without increasing the spall velocity.

Lastly, we observe microjets originating from the droplet center induced by the impact of the second pulse and find that the jet velocity increases with both an increase in interpulse spacing as well as the energy of the second pulse. The observed jetting and cavitation phenomena show strong resemblance to many cavitation and jetting studies in the available literature on transparent liquids, and provide an outlook to study the jet formation and behavior resulting from the interaction of in-dropletembedded vapor cavities with pressure waves.

Combined, our findings provide valuable insights into the complex processes resulting from laser impact on liquid tin microdroplets, enabling, e.g., a manipulation of the target expansion and spall characteristics using a doublepulse sequence. The present work further benefits the design of more advanced target-shaping pulse sequences in next-generation droplet-based extreme ultraviolet sources.

\section{ACKNOWLEDGMENTS}

We thank the anonymous reviewer for their highly constructive feedback. This work has been carried out 
at the Advanced Research Center for Nanolithography (ARCNL), a public-private partnership of the University of Amsterdam (UvA), the Vrije Universiteit Amsterdam (VU), the Netherlands Organisation for Scientific Research (NWO), and the semiconductor equipment manufacturer ASML.

[1] S. Fujioka, M. Shimomura, Y. Shimada, S. Maeda, H. Sakaguchi, Y. Nakai, T. Aota, H. Nishimura, N. Ozaki, A. Sunahara, K. Nishihara, N. Miyanaga, Y. Izawa, and K. Mima, Pure-tin microdroplets irradiated with double laser pulses for efficient and minimum-mass extreme-ultraviolet light source production, Appl. Phys. Lett. 92, 241502 (2008).

[2] I. V. Fomenkov, A. A. Schafgans, and D. Brandt, Laserproduced plasma sources for high-volume-Manufacturing EUV lithography, Synchrotron Radiat. News 32, 3 (2019).

[3] F. Torretti, J. Sheil, R. Schupp, M. M. Basko, M. Bayraktar, R. A. Meijer, S. Witte, W. Ubachs, R. Hoekstra, O. O. Versolato, A. J. Neukirch, and J. Colgan, Prominent radiative contributions from multiply-excited states in laserproduced tin plasma for nanolithography, Nat. Commun. 11, 2334 (2020).

[4] A. L. Klein, W. Bouwhuis, C. W. Visser, H. Lhuissier, C. Sun, J. H. Snoeijer, E. Villermaux, D. Lohse, and H. Gelderblom, Drop Shaping by Laser-Pulse Impact, Phys. Rev. Appl. 3, 044018 (2015).

[5] D. Kurilovich, A. L. Klein, F. Torretti, A. Lassise, R. Hoekstra, W. Ubachs, H. Gelderblom, and O. O. Versolato, Plasma Propulsion of a Metallic Microdroplet and its Deformation upon Laser Impact, Phys. Rev. Appl. 6, 014018 (2016).ArXiv:1604.00214.

[6] D. Kurilovich, M. M. Basko, D. A. Kim, F. Torretti, R. Schupp, J. C. Visschers, J. Scheers, R. Hoekstra, W. Ubachs, and O. O. Versolato, Power-law scaling of plasma pressure on laser-ablated tin microdroplets, Phys. Plasmas 25, 012709 (2018). ArXiv:1710.11426.

[7] H. Gelderblom, H. Lhuissier, A. L. Klein, W. Bouwhuis, D. Lohse, E. Villermaux, and J. H. Snoeijer, Drop deformation by laser-pulse impact, J. Fluid Mech. 794, 676 (2016).

[8] A. L. Klein, D. Kurilovich, H. Lhuissier, O. O. Versolato, D. Lohse, E. Villermaux, and H. Gelderblom, Drop fragmentation by laser-pulse impact, J. Fluid Mech. 893, A7(37) (2020). ArXiv:1910.02522.

[9] D. Hudgins, N. Gambino, B. Rollinger, and R. Abhari, Neutral cluster debris dynamics in droplet-based laser-produced plasma sources, J. Phys. D: Appl. Phys. 49, 185205 (2016).

[10] B. Liu, D. Kurilovich, H. Gelderblom, and O. O. Versolato, Mass Loss from a Stretching Semitransparent Sheet of Liquid Tin, Phys. Rev. Appl. 13, 024035 (2020).

[11] C. D. Ohl, Cavitation inception following shock wave passage, Phys. Fluids 14, 3512 (2002).

[12] M. S. Krivokorytov, Q. Zeng, B. V. Lakatosh, A. Y. Vinokhodov, Y. V. Sidelnikov, V. O. Kompanets, V. M. Krivtsun, K. N. Koshelev, C. D. Ohl, and V. V. Medvedev, Shaping and controlled fragmentation of liquid metal droplets through cavitation, Sci. Rep. 8, 597 (2018).
[13] A. Y. Vinokhodov, K. N. Koshelev, V. M. Krivtsun, M. S. Krivokorytov, Y. V. Sidelnikov, V. V. Medvedev, V. O. Kompanets, A. A. Melnikov, and S. V. Chekalin, Formation of a fine-dispersed liquid-metal target under the action of femto- and picosecond laser pulses for a laser-plasma radiation source in the extreme ultraviolet range, Quantum Elec. 46, 23 (2016).

[14] K. Tomita, Y. Sato, S. Tsukiyama, T. Eguchi, K. Uchino, K. Kouge, H. Tomuro, T. Yanagida, Y. Wada, M. Kunishima, G. Soumagne, T. Kodama, H. Mizoguchi, A. Sunahara, and K. Nishihara, Time-resolved two-dimensional profiles of electron density and temperature of laser-produced tin plasmas for extreme-ultraviolet lithography light sources, Sci. Rep. 7, 12328 (2017).

[15] M. S. Krivokorytov, A. Yu Vinokhodov, Y. V. Sidelnikov, V. M. Krivtsun, V. O. Kompanets, A. A. Lash, K. N. Koshelev, V. V. Medvedev, A. Y. Vinokhodov, Y. V. Sidelnikov, V. M. Krivtsun, V. O. Kompanets, A. A. Lash, K. N. Koshelev, and V. V. Medvedev, Cavitation and spallation in liquid metal droplets produced by subpicosecond pulsed laser radiation, Phys. Rev. E 95, 031101(R) (2017).

[16] M. M. Basko, M. S. Krivokorytov, A. Yu Vinokhodov, Y. V. Sidelnikov, V. M. Krivtsun, V. V. Medvedev, D. A. Kim, V. O. Kompanets, A. A. Lash, K. N. Koshelev, and A. Y. Vinokhodov, Fragmentation dynamics of liquid-metal droplets under ultra-short laser pulses, Laser Phys. Lett. 14, 036001 (2017).

[17] S. Y. Grigoryev, B. V. Lakatosh, M. S. Krivokorytov, V. V. Zhakhovsky, S. A. Dyachkov, D. K. Ilnitsky, K. P. Migdal, N. A. Inogamov, A. Y. Vinokhodov, V. O. Kompanets, Y. V. Sidelnikov, V. M. Krivtsun, K. N. Koshelev, and V. V. Medvedev, Expansion and Fragmentation of Liquid Metal Droplet by a Short Laser Pulse, Phys. Rev. Appl. 10, 064009 (2018). ArXiv:1807.01862.

[18] D. Kurilovich, T. D. F. Pinto, F. Torretti, R. Schupp, J. Scheers, A. S. Stodolna, H. Gelderblom, K. S. Eikema, S. Witte, W. Ubachs, R. Hoekstra, and O. O. Versolato, Expansion Dynamics After Laser-Induced Cavitation in Liquid Tin Microdroplets, Phys. Rev. Appl. 10, 054005 (2018). ArXiv:1805.07283.

[19] T. D. F. Pinto, J. Mathijssen, R. Meijer, H. Zhang, A. Bayerle, D. Kurilovich, O. O. Versolato, K. S. E. Eikema, and S. Witte, Cylindrically and non-cylindrically symmetric expansion dynamics of tin microdroplets after ultrashort laser pulse impact, Appl. Phys. A 127, 93 (2021).

[20] T. De Rességuier, L. Signor, A. Dragon, P. Severin, and M. Boustie, Spallation in laser shock-loaded tin below and just above melting on release, J. Appl. Phys. 102, 073535 (2007).

[21] M. A. Meyers and C. Taylor Aimone, Dynamic fracture (spalling) of metals, Prog. Mater. Sci. 28, 1 (1983).

[22] J. Fujimoto, T. Hori, T. Yanagida, and H. Mizoguchi, Development of laser-produced tin plasma-based EUV light source technology for HVM EUV lithography, Phys. Res. Int. 2012, 249495 (2012).

[23] Y. Tao, J. T. Stewart, J. Jur, A. LaForge, D. Brown, M. J. Arcand, A. A. Schafgans, and M. A. Purvis, Extreme ultraviolet light source, ASML Netherlands B.V., US Patent 2016/0007434 A1. 
[24] M. Purvis, I. V. Fomenkov, A. A. Schafgans, P. Mayer, K. Hummler, M. H. Leenders, Y. Tao, S. I. Rokitski, J. Stewart, A. I. Ershov, R. J. Rafac, S. De Dea, G. O. Vaschenko, D. C. Brandt, and D. J. Brown, in X-Ray Lasers and Coherent X-Ray Sources: Development and Applications XIII, edited by A. Klisnick and C. S. Menoni, International Society for Optics and Photonics (SPIE, San Diego, California, USA, 2019), Vol. 11111, p. 19.

[25] A. S. Stodolna, T. d. F. Pinto, F. Ali, A. Bayerle, D. Kurilovich, J. Mathijssen, R. Hoekstra, O. O. Versolato, K. S. Eikema, and S. Witte, Controlling ion kinetic energy distributions in laser produced plasma sources by means of a picosecond pulse pair, J. Appl. Phys. 124, 053303 (2018).

[26] R. A. Meijer, A. S. Stodolna, K. S. Eikema, and S. Witte, High-energy nd:YAG laser system with arbitrary subnanosecond pulse shaping capability, Opt. Lett. 42, 2758 (2017).

[27] A. E. Mayer and P. N. Mayer, Strain rate dependence of spall strength for solid and molten lead and tin, Int. J. Fracture 222, 171 (2020).

[28] M. M. Basko, On the theory and numerical modeling of spall fracture in pure liquids, arXiv:2102.01548 (2021).

[29] Y. B. Zel'Dovich and Y. P. Raizer, Physics of Shock Waves and High-Temperature Hydrodynamic Phenomena (Dover Publications, Mineola, New York, USA, 2002), Chap. XI.11, p. 716.

[30] C. A. Stan, P. R. Willmott, H. A. Stone, J. E. Koglin, M. Liang, A. L. Aquila, J. S. Robinson, K. L. Gumerlock, G. Blaj, R. G. Sierra, S. Boutet, S. A. Guillet, R. H. Curtis, S. L. Vetter, H. Loos, J. L. Turner, and F. J. Decker, Negative pressures and spallation in water drops subjected to nanosecond shock waves, J. Phys. Chem. Lett. 7, 2055 (2016).

[31] M. J. Assael, A. E. Kalyva, K. D. Antoniadis, R. Michael Banish, I. Egry, J. Wu, E. Kaschnitz, and W. A. Wakeham, Reference data for the density and viscosity of liquid copper and liquid tin, J. Phys. Chem. Ref. Data 39, 033105 (2010).

[32] P. W. Humrickhouse, An equation of state and compendium of thermophysical properties of liquid tin, a prospective plasma-facing material, IEEE Trans. Plasma Sci. 47, 3374 (2019).

[33] D. Grady, Physics of Shock and Impact: Volume 1, Fundamentals and Dynamic Failure (IOP Publishing, 2017).

[34] M. M. Basko, J. Maruhn, and A. Tauschwitz, An efficient cell-centered diffusion scheme for quadrilateral grids, J. Comput. Phys. 228, 2175 (2009).

[35] M. M. Basko, J. Maruhn, and A. Tauschwitz, Development of a 2D Radiation-Hydrodynamics Code RALEF for Laser Plasma Simulations GSI Report 2010-1, PlasmaPhysics 25, 410 (GSI Helmholtzzentrum flur Schwerionenforschung $\mathrm{GmbH}, 2010$ ).

[36] M. M. Basko, P. V. Sasorov, M. Murakami, V. G. Novikov, and A. S. Grushin, One-dimensional study of the radiationdominated implosion of a cylindrical tungsten plasma column, Plasma Phys. Controlled Fusion 54, 055003 (2012).

[37] M. M. Basko, V. G. Novikov, and A. S. Grushin, On the structure of quasi-stationary laser ablation fronts in strongly radiating plasmas, Phys. Plasmas 22, 053111 (2015).
[38] M. M. Basko, On the maximum conversion efficiency into the 13.5-nm extreme ultraviolet emission under a steadystate laser ablation of tin microspheres, Phys. Plasmas 23, 083114 (2016).

[39] J. R. Blake and D. C. Gibson, Growth and collapse of A vapour cavity near A free surface, J. Fluid Mech. 111, 123 (1981).

[40] W. Lauterborn and C. D. Ohl, Cavitation bubble dynamics., Ultrason. Sonochem. 4, 65 (1997).

[41] P. B. Robinson, J. R. Blake, T. Kodama, A. Shima, and Y. Tomita, Interaction of cavitation bubbles with a free surface, J. Appl. Phys. 89, 8225 (2001).

[42] D. Obreschkow, P. Kobel, N. Dorsaz, A. De Bosset, C. Nicollier, and M. Farhat, Cavitation Bubble Dynamics inside Liquid Drops in Microgravity, Phys. Rev. Lett. 97, 094502 (2006).

[43] S. T. Thoroddsen, K. Takehara, T. G. Etoh, and C. D. Ohl, Spray and microjets produced by focusing a laser pulse into a hemispherical drop, Phys. Fluids 21, 112101 (2009).

[44] C. D. Ohl and R. Ikink, Shock-Wave-Induced Jetting of Micron-Size Bubbles, Phys. Rev. Lett. 90, 214502 (2003).

[45] E. Klaseboer, S. W. Fong, C. K. Turangan, B. C. Khoo, J. Andrew, M. L. Calvisi, G. N. Sankin, and P. Zhong, Interaction of lithotripter shockwaves with single inertial cavitation bubbles, J. Fluid. Mech. 593, 33 (2007).

[46] A. Antkowiak, N. Bremond, S. Le Dizès, and E. Villermaux, Short-term dynamics of a density interface following an impact, J. Fluid Mech. 577, 241 (2007).

[47] G. J. Ball, B. P. Howell, T. G. Leighton, and M. J. Schofield, Shock-induced collapse of a cylindrical air cavity in water: A free-lagrange simulation, Shock Waves 10, 265 (2000).

[48] J. F. Haas, Interaction of weak shock waves with cylindrical and spherical gas inhomogeneities, J. Fluid Mech. 181, 41 (1987).

[49] G. N. Sankin, W. N. Simmons, S. L. Zhu, and P. Zhong, Shock Wave Interaction with Laser-Generated Single Bubbles, Phys. Rev. Lett. 95, 034501 (2005).

[50] I. R. Peters, Y. Tagawa, N. Oudalov, C. Sun, A. Prosperetti, D. Lohse, and D. Van Der Meer, Highly focused supersonic microjets: Numerical simulations, J. Fluid Mech. 719, 587 (2013). ArXiv:1203.5029.

[51] G. Xiang and B. Wang, Numerical study of a planar shock interacting with a cylindrical water column embedded with an air cavity, J. Fluid Mech. 825, 825 (2017).

[52] Y. Zhu, Z. Yang, K. H. Luo, J. Pan, and Z. Pan, Numerical investigation of planar shock wave impinging on spherical gas bubble with different densities, Phys. Fluids 31, 056101 (2019).

[53] Y. Liang, Y. Jiang, C. Y. Wen, and Y. Liu, Interaction of a planar shock wave and a water droplet embedded with a vapour cavity, J. Fluid Mech. 885, R6(13) (2020).

[54] D. Hudgins, Ph.D. thesis, ETH Zürich, 2019.

[55] S. R. G. Avila and C. D. Ohl, Fragmentation of acoustically levitating droplets by laser-induced cavitation bubbles, J. Fluid Mech. 805, 551 (2016).

[56] A. Philipp, C. Scheffczyk, and A. Vogel, Interaction of lithotripter-generated shock waves with air bubbles, J. Acoustical Soc. Am. 93, 2496 (1993). 